\title{
Metal Coordination Is Crucial for Geranylgeranyl Diphosphate Synthase-Bisphosphonate Interactions: A Crystallographic and Computational Analysis $\$$
}

\author{
Michal Lisnyansky, Elon Yariv, Omri Segal, Milit Marom, Anat Loewenstein, Nir Ben-Tal, \\ Moshe Giladi, and Yoni Haitin \\ Department of Physiology and Pharmacology, Sackler Faculty of Medicine (M.L., M.M., M.G., Y.H.), Department of Biochemistry \\ and Molecular Biology, George S. Wise Faculty of Life Sciences (E.Y., N.B.-T.), and Sackler Faculty of Medicine (O.S., A.L.), Tel \\ Aviv University, Tel Aviv, Israel; Ophthalmology Division, Tel Aviv Sourasky Medical Center, Tel Aviv, Israel (A.L.); and Tel Aviv \\ Sourasky Medical Center, Tel Aviv, Israel (M.G.)
}

Received June 7, 2019; accepted August 10, 2019

\begin{abstract}
Geranylgeranyl diphosphate synthase (GGPPS) is a central metalloenzyme in the mevalonate pathway, crucial for the prenylation of small GTPases. As small GTPases are pivotal for cellular survival, GGPPS was highlighted as a potential target for treating human diseases, including solid and hematologic malignancies and parasitic infections. Most available GGPPS inhibitors are bisphosphonates, but the clinically available compounds demonstrate poor pharmacokinetic properties. Although the design of novel bisphosphonates with improved physicochemical properties is highly desirable, the structure of wild-type human GGPPS (hGGPPS) bound to a bisphosphonate has not been resolved. Moreover, various metalbisphosphonate-binding stoichiometries were previously reported in structures of yeast GGPPS (yGGPPS), hampering computational drug design with metal-binding pharmacophores (MBP). In this study, we report the $2.2 \AA$ crystal structure of hGGPPS in complex with ibandronate, clearly depicting the
\end{abstract}

involvement of three $\mathrm{Mg}^{2+}$ ions in bisphosphonate-protein interactions. Using drug-binding assays and computational docking, we show that the assignment of three $\mathrm{Mg}^{2+}$ ions to the binding site of both hGGPPS and yGGPPS greatly improves the correlation between calculated binding energies and experimentally measured affinities. This work provides a structural basis for future rational design of additional MBP-harboring drugs targeting hGGPPS.

\section{SIGNIFICANCE STATEMENT}

Bisphosphonates are inhibitors of geranylgeranyl diphosphate synthase (GGPPS), a metalloenzyme crucial for cell survival. Bisphosphonate binding depends on coordination by $\mathrm{Mg}^{2+}$ ions, but various $\mathrm{Mg}^{2+}$-bisphosphonate-binding stoichiometries were previously reported. In this study, we show that three $\mathrm{Mg}^{2+}$ ions are vital for drug binding and provide a structural basis for future computational design of GGPPS inhibitors.

\section{Introduction}

Geranylgeranyl diphosphate (GGPP, $\mathrm{C}_{20}$ ) synthase (GGPPS) is an important mevalonate pathway trans-prenyltransferase (Liang et al., 2002). By catalyzing the condensation of isopentenyl pyrophosphate with farnesyl diphosphate (FPP, $\mathrm{C}_{15}$ ), GGPPS synthesizes GGPP, used for post-translational protein prenylation (Wang and Casey, 2016) (Fig. 1). Intriguingly,

This work was supported by Israel Science Foundation [Grants 1721/16 and 1775/12 to Y.H.], Israel Cancer Research Foundation [Grant 01214 to Y.H.], German-Israeli Foundation [Grant I-2425-418.13/2016 to Y.H.], Shtacher Foundation (to Y.H. and M.G.), Tel-Aviv Sourasky Medical Center (A.L. and M.G.), and Clair and Amedee Maratier Institute for the Study of Blindness and Visual Disorders, Sackler Faculty of Medicine, Tel-Aviv University (Y.H.)

The authors declare that no conflict of interest exists.

https://doi.org/10.1124/mol.119.117499.

S This article has supplemental material available at molpharm. aspetjournals.org. although the fungal, bacterial, and plant orthologs exhibit dimeric structures, human GGPPS (hGGPPS) forms a hexamer composed of three dimers (Kavanagh et al., 2006a).

Protein prenylation is crucial for the membrane association and function of proteins essential for cellular survival. Importantly, most small GTPases, including pivotal oncogenes such as RAS and RHO, require prenylation for their normal function (Bourne et al., 1990; Konstantinopoulos et al., 2007), making inhibition of this process an attractive therapeutic target. Indeed, bisphosphonates are a group of drugs that induce cellular apoptosis by inhibiting isoprenoid biosynthesis (Kavanagh et al., 2006b). Currently, bisphosphonates are used to treat osteoporosis, a common skeletal disorder (Russell, 2011). By specifically incorporating into the bone matrix, the drug is released upon resorption by osteoclasts, undergoes endocytosis, and inhibits GGPPS, along with the upstream enzyme FPP synthase (Guo et al.,

ABBREVIATIONS: CN, coordination number; FPP, farnesyl diphosphate; GGPP, geranylgeranyl diphosphate; GGPPS, GGPP synthase; hGGPPS, human GGPPS; MBP, metal-binding pharmacophore; PDB, Protein Data Bank; TCEP, tris(2-carboxyethyl)phosphine; TEV, tobacco etch virus; WT, wild-type; yGGPPS, yeast GGPPS. 

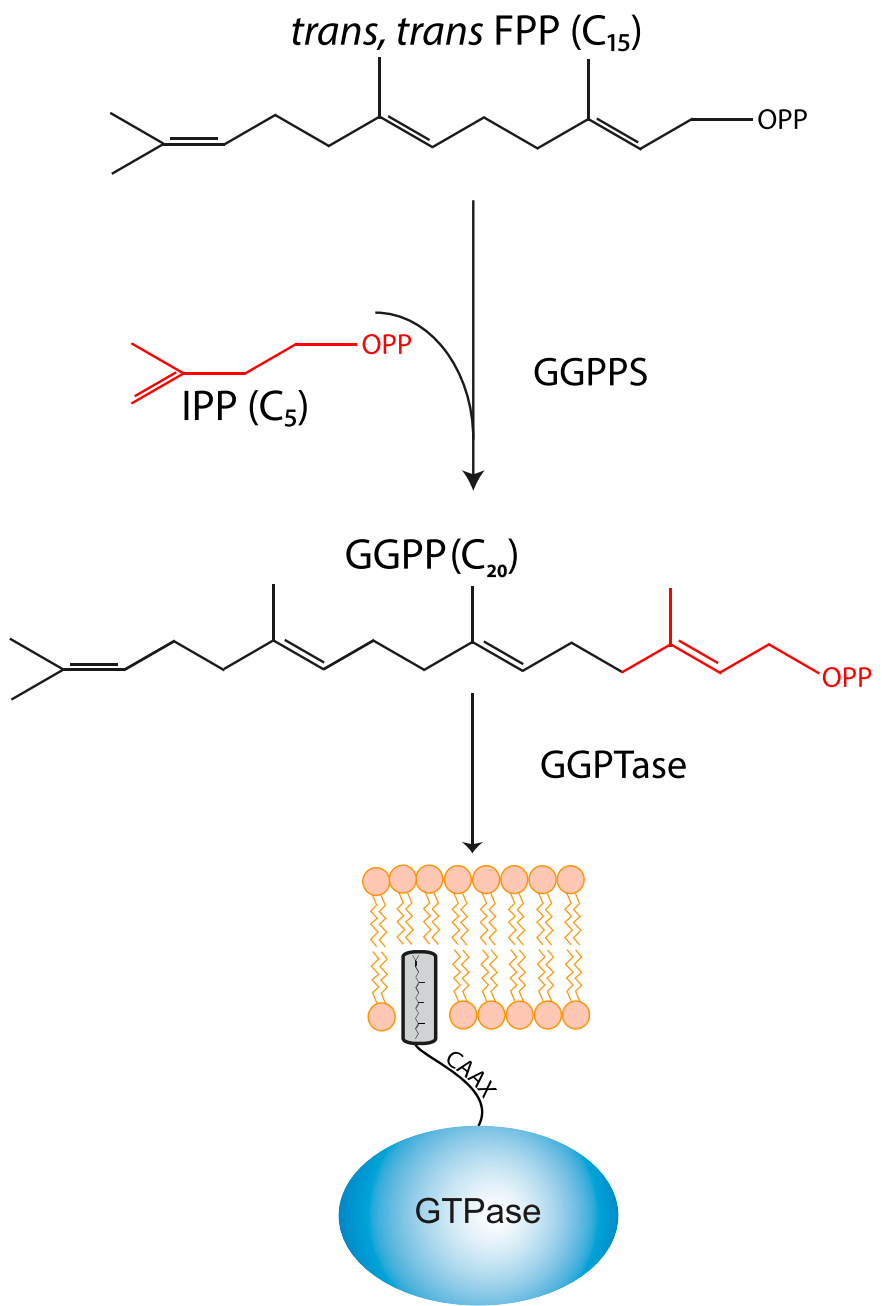

Fig. 1. Catalytic cycle of GGPPS. FPP $\left(\mathrm{C}_{15}\right)$ and isopentenyl diphosphate $\left(\mathrm{C}_{5}\right)$ are condensed by GGPPS to form GGPP $\left(\mathrm{C}_{20}\right)$. The geranylgeranyl moiety is then transferred from GGPP to a C-terminal CAAX motif (C-cysteine, A-aliphatic residue, X-leucine/phenylalanine) by GGPTase (geranylgeranyl protein transferase), resulting in membrane association of small GTPases.

2007; Russell, 2011). The resultant decrease in small GTPases prenylation leads to osteoclasts apoptosis and decreased bone resorption (Russell, 2011).

The profound cellular alterations induced by inhibition of protein prenylation, and specifically, by a decrease in prenylation of small GTPases (Russell, 2011), highlighted GGPPS as a potential target for cancer therapy (Downward, 2003; Jiang et al., 2014; Mullen et al., 2016; Lacbay et al., 2018). Moreover, GGPPS enzymes from parasites leading to human diseases are considered as potential drug targets to inhibit parasitic growth in vitro and in vivo (Szabo et al., 2002; G Ricci et al., 2016). However, the physicochemical properties of the marketed bisphosphonates render them with poor bioavailability and cell permeability characteristics (Lin, 1996), restricting their therapeutic scope for treatment of bone disorders, in which the drug accumulates locally and permeates the cell membrane via endocytosis during bone resorption (Russell, 2011). Thus, to exploit the full potential of GGPPS inhibition, the design of novel bisphosphonates with modified pharmacokinetic characteristics is required for expansion of their target tissues repertoire (Haney et al., 2017).
Over the past two decades, several attempts were made to establish robust computational screening approaches to predict the binding potency of bisphosphonates to GGPPS (Szabo et al., 2002; Guo et al., 2007; Haney et al., 2017). These attempts were based on the wealth of structural information obtained using the yeast GGPPS (yGGPPS) ortholog bound to various bisphosphonates (Guo et al., 2007; K-M Chen et al., 2008; Zhang et al., 2009), despite differences in the potency of these drugs toward yGGPPS and hGGPPS (Guo et al., 2007), as the structure of hGGPPS-wild-type (WT) bound to a bisphosphonate was unavailable.

We have recently determined the crystal structure of hGGPPS-D188Y, involved in bisphosphonate-induced atypical fractures, in complex with zoledronate (Lisnyansky et al., 2018). Our analysis revealed the involvement of three $\mathrm{Mg}^{2+}$ ions in GGPPS-bisphosphonate interaction, a metal-ligand stoichiometry deviating from that observed in most previously determined yGGPPS structures (Lisnyansky et al., 2018). Bisphosphonates harbor a metal-binding pharmacophore (MBP) that allows strong protein-ligand interactions mediated by coordinated metal ions (Cohen, 2017; Jiang et al., 2019). However, the use of such MBPs in drug design requires accurate positioning of metal ions in the binding site (Harding et al., 2010; Handing et al., 2018). Importantly, the assignment of $\mathrm{Mg}^{2+}$ ions, involved in GGPPSbisphosphonate interactions, has proven to be especially challenging (Nayal and Di Cera, 1996; Zheng et al., 2008).

The discrepancy in the metal-ligand interaction network observed in the mutant hGGPPS, compared with previously determined yGGPPS structures, yields profound ramifications on computational drug screening and design attempts due to the different electrostatic and steric properties of the binding pocket. Indeed, exploration of the chemical space in the design of metalloenzyme inhibitors greatly depends on metal-ligand interactions (Riccardi et al., 2018). Moreover, molecular docking taking into account metal-ligand interactions has proven successful in obtaining potent and specific metalloenzyme inhibitors (Bruno et al., 2017). Therefore, we have determined the first crystal structure of hGGPPS-WT in complex with the bisphosphonate ibandronate. Furthermore, to explore possible ortholog-dependent structural differences in metal-ligand coordination, we have reanalyzed eight previously solved structures of yGGPPS in complex with various bisphosphonates and assigned previously misidentified $\mathrm{Mg}^{2+}$ ions to the bisphosphonate binding site. Using a conjunction of drug-binding assays and computational docking, we show the essential role of $\mathrm{Mg}^{2+}$ ions stoichiometry in GGPPSbisphosphonate interaction and the contribution of metal-ligand interactions to the predictivity of computational models for GGPPS inhibitors.

\section{Materials and Methods}

Overexpression and Purification of hGGPPS. cDNA of fulllength human GGPS1 (transOMIC, Huntsville, AL) was cloned into pETM-11 vector with N-terminal hexahistidine tag, followed by a tobacco etch virus (TEV) protease cleavage site. Proteins were overexpressed and purified, as previously described (Kavanagh et al., 2006a; Lisnyansky et al., 2018), using immobilized metal affinity chromatography and size-exclusion chromatography. Briefly, Escherichia coli $\mathrm{T} 7$ express competent cells, transformed with the GGPPS construct, were grown in Terrific Broth medium at $37^{\circ} \mathrm{C}$ until reaching optical density $600 \mathrm{~nm}=0.6$ and induced at $16^{\circ} \mathrm{C}$ by adding $0.5 \mathrm{mM}$ 
isopropyl $\beta$-D-1-thiogalactopyranoside. Proteins were expressed at $16^{\circ} \mathrm{C}$ for 16 to 20 hours and harvested by centrifugation $(10,000 \mathrm{~g}$ for 10 minutes). Cells were suspended in buffer A, containing $50 \mathrm{mM}$ HEPES, pH 7.5, $500 \mathrm{mM} \mathrm{NaCl}, 5 \%$ glycerol, $0.5 \mathrm{mM}$ tris(2-carboxyethyl)phosphine (TCEP), $1 \mu \mathrm{g} / \mathrm{ml}$ DNase I, $1 \mathrm{mM}$ phenylmethane sulfonyl fluoride, and Protease Inhibitor Cocktail Set III (Calbiochem, San Diego, CA). The cells were disrupted in an EmulsiFlex C3 homogenizer (Avestin, Ottawa, ON, Canada). The soluble protein was then recovered by centrifugation at $\sim 32,000 \mathrm{~g}$ for 45 minutes at $4^{\circ}$ C. The supernatant was loaded onto a $\mathrm{Ni}^{2+}$-nitrilotriacetic acid column, followed by thorough washing with buffer A, supplemented with $30 \mathrm{mM}$ imidazole to reduce nonspecific protein binding. Next, overexpressed proteins were eluted with buffer A supplemented with $250 \mathrm{mM}$ imidazole. Imidazole was removed using a HiPrep 26/10 desalting column equilibrated with buffer $\mathrm{A}$, followed by the addition of $6 \times$ His-tagged TEV protease $(1 \mathrm{mg}$ TEV protease per $50 \mathrm{mg}$ protein) to the eluate, to remove their $6 \times$ His-tagged, at $4^{\circ} \mathrm{C}$ overnight. The cleaved proteins were then loaded again onto a $\mathrm{Ni}^{2+}$-nitrilotriacetic acid column pre-equilibrated with buffer A containing $30 \mathrm{mM}$ imidazole to remove the cleaved $6 \times$ His-tag and TEV protease. The flowthrough was collected, concentrated to 3 to $4 \mathrm{ml}$, and loaded onto a HiLoad 16/60 Superdex-200 column (GE healthcare, UK) equilibrated with $10 \mathrm{mM}$ HEPES, pH 7.5, $100 \mathrm{mM} \mathrm{NaCl}, 1 \mathrm{mM} \mathrm{MgCl}$, and $0.5 \mathrm{mM}$ TCEP for final purification. Purified proteins were concentrated, flash-frozen in liquid nitrogen, and stored at $-80^{\circ} \mathrm{C}$.

Crystallization and Structure Determination of Ibandronate-Bound hGGPPS. Initial crystallization screens were performed using $38 \mathrm{mg} / \mathrm{ml}$ purified hGGPPS at $19^{\circ} \mathrm{C}$ using the sitting drop vapor diffusion method. Initial crystals were obtained in $0.1 \mathrm{M}$ sodium formate, $\mathrm{pH} 7.0,12 \% \mathrm{w} / \mathrm{v}$ PEG 3350. Crystals were soaked overnight with mother liquor solution supplemented with $350 \mu \mathrm{M}$ ibandronate, cryoprotected with $20 \%$ glycerol, and immersed in liquid $\mathrm{N}_{2}$. Data were collected at $100^{\circ} \mathrm{K}$ at the European Synchrotron Radiation Facility (Grenoble, France). Integration, scaling, and merging of the diffraction data were done with the XDS program (Kabsch, 2010). As the data were slightly anisotropic, an ellipsoidal truncation and anisotropic scaling were performed (Strong et al., 2006) using resolution cutoffs of $\mathrm{a}^{*}=2.3 \AA, \mathrm{b}^{*}=2.2 \AA$, and $\mathrm{c}^{*}=2.2 \AA$. The structure was solved by molecular replacement using the programs PHASER (McCoy et al., 2007) and PHENIX (Adams et al., 2010) (Table 1). The structure of a single dimer from GGPPS-WT [Protein Data Bank (PDB) 2Q80] was used as a search model. Iterative model building and refinement were carried out in PHENIX with manual adjustments using COOT (Emsley and Cowtan, 2004). Structural illustrations were prepared with University of California, San Francisco Chimera (https://www.cgl.ucsf.edu/chimera) or PyMOL (https://pymol.org). Atomic coordinates and structure factors for the structure of hGGPPS in complex with ibandronate have been deposited in the PDB with accession number $6 \mathrm{R} 4 \mathrm{~V}$.

Assignment of $\mathrm{Mg}^{2+}$ Ions. To assign $\mathrm{Mg}^{2+}$ ions, we have used several restrains, including the average bond lengths, coordination number $(\mathrm{CN})$, and the identity of the oxygen donors. The usual $\mathrm{CN}$ for $\mathrm{Mg}^{2+}$ in proteins is six, with one to two protein donor oxygen atoms and the rest arising from phosphate groups and water molecules. However, incomplete coordination spheres in medium resolution structures (2-2.5 $)$, as the available structures of yGGPPS, are commonly observed. Therefore, we used the average CNCN (4.5 \pm 1.5) in medium-resolution structures (Zheng et al., 2008) and considered binding sites with $\mathrm{CN}=3-6$. In addition, although based on the Cambridge Structural Database (Kennard, 2015), the average $\mathrm{Mg}^{2+}$-O bond length is $2.16 \AA$, in the given resolution range, and using a cutoff of $3 \AA$ to define the coordination sphere, the mean $\mathrm{Mg}^{2+}-\mathrm{O}$ bond length is $2.24 \pm 0.26 \AA$ with a complete coordination sphere [coordination number $(\mathrm{CN}) \geq 5$ ] and $2.37 \pm 0.32 \AA$ with an incomplete coordination sphere $(\mathrm{CN}<5)$ (Zheng et al., 2008). These bond length values were used as reference in the current analysis.

Differential Scanning Fluorimetry. Thermal shift assay was performed using a real-time polymerase chain reaction system
TABLE 1

Crystallographic statistics for hGGPPS in complex with ibandronate

\begin{tabular}{|c|c|}
\hline \multicolumn{2}{|l|}{ Data Collection } \\
\hline Space group & $\mathrm{P} 2_{1} 2_{1} 2_{1}$ \\
\hline Cell dimensions & \\
\hline $\mathrm{a}, \mathrm{b}, \mathrm{c}(\AA)$ & $68.40,152.80,198.40$ \\
\hline$\alpha, \beta, \gamma\left({ }^{\circ}\right)$ & $90,90,90$ \\
\hline Beamline & ESRF ID30B \\
\hline Wavelength (̊̊) & 0.976 \\
\hline Resolution $(\AA)$ & 2.2 \\
\hline Multiplicity & 6.58 \\
\hline Completeness (\%) & 99.8 \\
\hline Mean $\mathrm{I} / \sigma(\mathrm{I})$ & 8.51 \\
\hline $\mathrm{R}_{\text {meas }}(\%)$ & 17.1 \\
\hline \multicolumn{2}{|l|}{ Refinement statistics } \\
\hline No. reflections (work/free) ${ }^{a}$ & $98,591 / 5215$ \\
\hline Resolution range $^{a}$ & 49.66-2.20 \\
\hline $\mathrm{R}_{\text {work }} / \mathrm{R}_{\text {free }}$ & $0.1787 / 0.2245$ \\
\hline \multicolumn{2}{|l|}{ No. atoms } \\
\hline Macromolecules & 13,678 \\
\hline Ligands & 143 \\
\hline Solvent & 1242 \\
\hline \multicolumn{2}{|l|}{ Average $B$-factor } \\
\hline Macromolecules & 36.3 \\
\hline Ligands & 51.0 \\
\hline Solvent & 43.0 \\
\hline RMSD (bond lengths) & 0.005 \\
\hline RMSD (bond angles) & 0.8 \\
\hline Ramachandran outliers (\%) & 0 \\
\hline
\end{tabular}

ESRF, European Synchrotron Radiation Facility; RMSD, root-mean-square deviation.

${ }^{a}$ After anisotropic scaling.

(Thermo Fisher Scientific, Waltham, MA) with the fluorescent dye SYPRO Orange (Thermo Fisher Scientific) using the ROX filter set in clear 96-well plates (Niesen et al., 2007). The temperature was increased using a continuous ramp at a rate of $1^{\circ} \mathrm{C} / \mathrm{min}$ from $25^{\circ} \mathrm{C}$ to $95^{\circ} \mathrm{C}$. Assays were performed in a final volume of $25 \mu \mathrm{l}$, containing $5 \mu \mathrm{M}$ protein, $5 \mathrm{X}$ SYPRO Orange, $100 \mathrm{mM} \mathrm{NaCl}, 10 \mathrm{mM}$ Na-HEPES, $1 \mathrm{mM} \mathrm{MgCl} 2,0.5 \mathrm{mM}$ TCEP, $\mathrm{pH} 7.5$, and different bisphosphonate concentrations $(0.25-500 \mu \mathrm{M})$. Melting temperatures were extracted by Boltzmann sigmoidal fitting of the ascending phase of the curves. Bisphosphonates-concentration dependence of the meltingmn temperature was fit using the Hill equation (Origin 7.0; OriginLab, Northampton, MA).

Computational Drug Docking. Coordinate files were prepared for molecular docking using the protein preparation wizard (Sastry et al., 2013) (Schrödinger, New York, NY). The protocol includes three stages, as follows: 1) addition of explicit hydrogens, bond order assignment, and building missing amino acid side chains; 2) determination of protonation state for protein side chains using Propka 3.1 (Olsson et al., 2011; Søndergaard et al., 2011); and 3) restrained energy minimization with 0.3 root-mean-square deviation cutoff for the heavy atoms. Ligprep (Schrödinger, New York, NY) was used to prepare the known inhibitors of GGPPS for molecular docking. Ionization states of ligands were determined at $\mathrm{pH}==7.0 \pm 2.0$ using Epik 4.0 (Greenwood et al., 2010). In addition to the normal protonation states, Epik generated metal-binding states for each ligand. Molecular docking was performed using Glide 7.5 (Friesner et al., 2004; Halgren et al., 2004). The receptor grid was generated around the geometric center of the ligand found in the crystal structures, with $10 \AA$ edges for the enclosing box. Ligands were docked with flexible conformer sampling and the standard precision-scoring function. The bisphosphonate core was restricted to the coordinates found in the crystal structure, with $0.5 \AA$ tolerance for deviations. For each ligand, up to 10 different poses were generated, the pose with the lowest root-mean-square deviation in comparison with the crystalized ligand was selected, and binding energy was estimated with molecular mechanics combined with the generalized BornBB and surface area continuum solvation method. The above method calculates binding 
energy using the following equation: $\Delta \mathrm{G}_{\text {binding }}=\mathrm{G}_{\text {complex }}-\mathrm{G}_{\text {ligand }}-$ $\mathrm{G}_{\text {protein. }}$. The internal energy is calculated using the molecular mechanics OPLS3 forcefield (Harder et al., 2016), whereas the desolvation energy and electrostatic interactions are calculated using Variable Dielectric and Surface Generalized Born (Li et al., 2011). To account for the differences between Glide's scoring function and molecular mechanics combined with the generalized Born and surface area continuum solvation method energies, the ligand pose was optimized using energy minimization prior to the binding energy determination. Correlations between the calculated binding energies and the experimentally obtained values were calculated using the Pearson correlation coefficient.

\section{Results}

Crystal Structure of hGGPPS in Complex with a Bisphosphonate. Bisphosphonates are used clinically in humans mainly for the treatment of osteoporosis (Russell, 2011). Although bisphosphonates are known to inhibit GGPPS, the structure of WT hGGPPS-bisphosphonate complex remained unresolved (Lisnyansky et al., 2018). Importantly, the main molecular blueprint for designing novel bisphosphonate for GGPPS inhibition is the wellcharacterized yGGPPS ortholog. Nevertheless, it was shown that bisphosphonates exhibit different affinities toward hGGPPS and yGGPPS, and their potency distributions differ for each enzyme (Guo et al., 2007). Interestingly, in some cases the affinities differ by a factor of 20 between the enzymes, whereas in other cases, the affinities are practically identical. Therefore, to unveil the molecular details of hGGPPS-bisphosphonate interaction, we determined the 2.2 A resolution crystal structure of hGGPPS in the presence of ibandronate ( Fig. 2; Supplemental Fig. 1; Supplemental Material 1; Table 1).

Similar to the previously reported structure of hGGPPS bound to GGPP (PDB 2Q80), the biologic assembly consists of a hexamer, composed of a trimer of dimers (Fig. 2A) (Kavanagh et al., 2006a). Moreover, the structures share high similarity, as reflected by a low root-mean-square deviation value $(0.63 \AA)$ for the entire hexameric assembly, indicating that the binding of ibandronate does not induce major conformational transitions. Within the dimers, each monomer exhibits the canonical trans-prenyltransferase fold, composed of $13 \alpha$-helices. Ten of the $13 \alpha$-helices surround a large central cavity, where the aspartate-rich conserved sequence tandem, forming the FPP and bisphosphonate binding sites, are localized (Liang et al., 2002).

Focusing on the bisphosphonate binding sites, we could identify three $\mathrm{Mg}^{2+}$ ions in five of six chains and two $\mathrm{Mg}^{2+}$ ions in one chain (Fig. 2B; Supplemental Fig. 2). This is in contrast to the coordination of GGPP, which includes only two $\mathrm{Mg}^{2+}$ ions (Kavanagh et al., 2006a). The $\mathrm{Mg}^{2+}$ ions in the hGGPPS-ibandronate structure are well coordinated, with an average $\mathrm{Mg}^{2+}-\mathrm{O}$ bond length of $2.19 \pm 0.21 \AA$ and a $\mathrm{CN}$ of $5.7 \pm 0.6$ (range 4-6) for the entire hexamer. The Mg1 site is coordinated by two oxygens from the phosphate group, two carboxyl oxygens from D64 and D68, and two water molecules (Fig. 2C). The Mg2 site is coordinated by one oxygen from the phosphate group, two carboxyl oxygens from D64 and D68, and three water molecules (Fig. 2D). The Mg3 site is
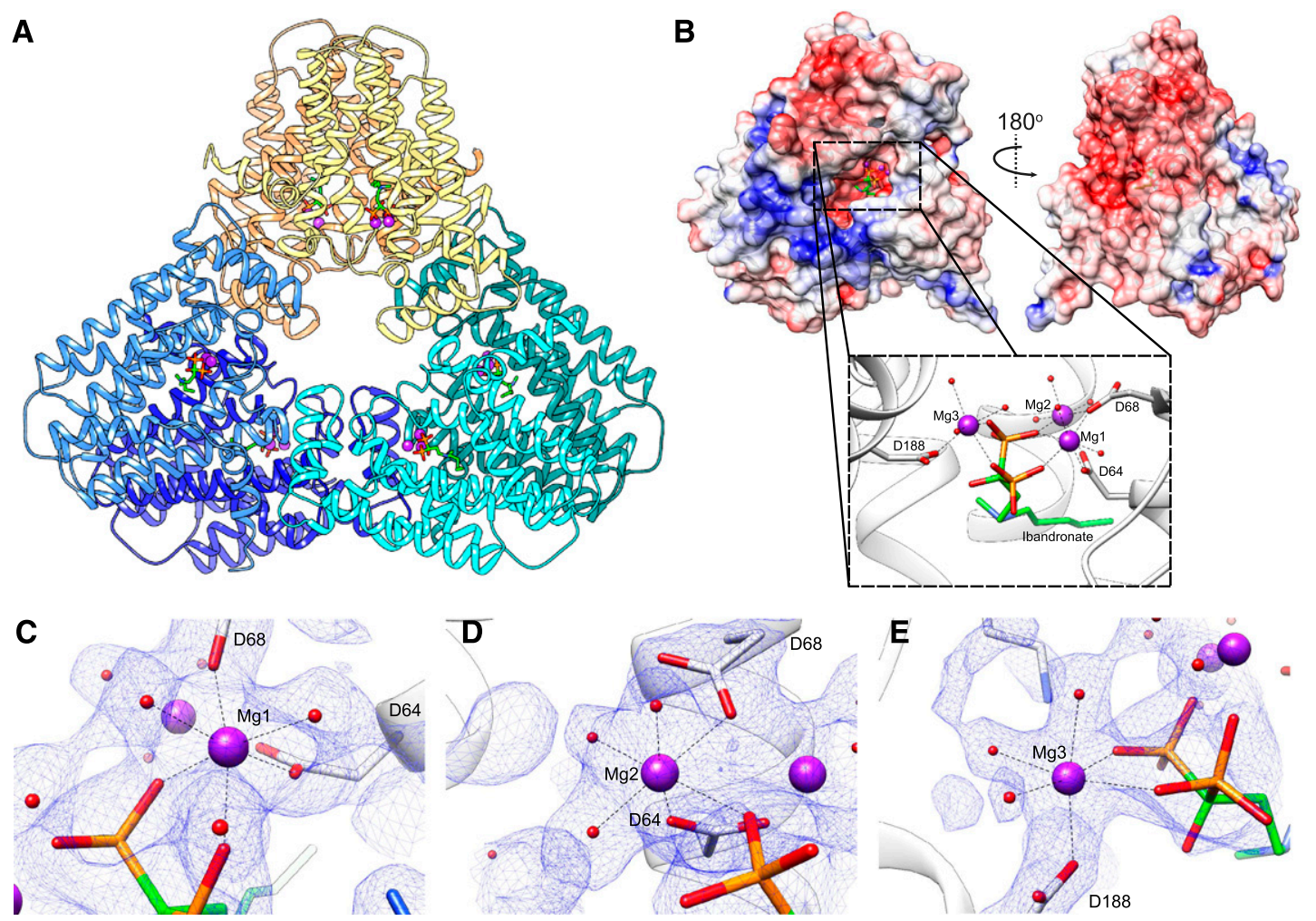

Fig. 2. Crystal structure of hGGPPS in complex with ibandronate. (A) The asymmetric unit, containing the biologic hexameric arrangement of ibandronate-bound hGGPPS, in cartoon representation. (B) Surface representation of one monomer colored according to the adaptive Poisson-Boltzmann solver surface electrostatics. The rectangle frames a zoom perspective of the ibandronate-binding pocket. Ibandronate and residues interacting with the $\mathrm{Mg}^{2+}$ ions (purple spheres) are shown as sticks. Water molecules are shown as red spheres. (C-E) The three Mg ${ }^{2+}$ binding sites, $\mathrm{Mg}_{1}$ (C), Mg2 (D), and Mg3 (E), are shown with their electron density contoured at 1.3 (blue mesh). 
coordinated by two oxygens from the phosphate group, one carboxyl oxygen from D188, and three water molecules (Fig. 2E). Overall, each $\mathrm{Mg}^{2+}$ ion is coordinated by one to two protein donors, one to two phosphate group donors, and two to three water molecules, matching the coordination observed in most $\mathrm{Mg}^{2+}$-protein complexes (Zheng et al., 2008; Harding et al., 2010). In summary, the first crystal structure of a hGGPPS-bisphosphonate complex clearly supports the notion that three $\mathrm{Mg}^{2+}$ ions are involved in the coordination of bisphosphonates by hGGPPS.

Effect of $\mathrm{Mg}^{2+}$ Ion Stoichiometry on Drug Docking to hGGPPS. Next, we sought to assess the predictivity of our hGGPPS-ibandronate structure as a model for computational drug screening, and to determine the significance of $\mathrm{Mg}^{2+}$ ions in hGGPPS-bisphosphonate interactions. To that end, we surveyed clinically available bisphosphonates and predicted their binding energies to the hGGPPS structure with $\mathrm{Mg}^{2+}$ ions occupying all three sites (Table 2). To assess the contribution of $\mathrm{Mg} 3$, which was not resolved in one of the chains and is also absent in several yGGPPS structures (Guo et al., 2007) (see below), we also performed this analysis with $\mathrm{Mg}^{2+}$ ions occupying only sites Mg1 and Mg2 (Table 2). Next, we used our previously described thermal shift assay to measure the binding affinities of six commercially available bisphosphonates to hGGPPS (Fig. 3A; Supplemental Fig. 3; Table 2) (Lisnyansky et al., 2018). Strikingly, in the presence of all three $\mathrm{Mg}^{2+}$ ions, the calculated binding energies are highly correlated with the measured $K_{d}$ values $\left(R^{2}=0.93\right.$, $P=0.002$, Fig. 3B). In sharp contrast, removal of the $\mathrm{Mg}^{2+}$ ion from the Mg3 site resulted in deterioration of the models predictivity, resulting in poor correlation of the calculated binding energies with the measured $K_{d}$ values $\left(R^{2}=0.33\right.$, $P=0.23$, Fig. $3 \mathrm{C}$ ).

Notably, the range of the measured $\mathrm{K}_{\mathrm{d}}$ values obtained for the commercially available compounds is rather narrow. Thus, we expanded our analysis by correlating previously reported $\mathrm{IC}_{50}$ values (Szabo et al., 2002), spanning three orders of magnitude, with calculated binding energies in the presence of three or two $\mathrm{Mg}^{2+}$ ions (Fig. 4A; Table 3). Again, a statistically significant correlation was obtained in the presence of all three $\mathrm{Mg}^{2+}$ ions between the calculated binding energies and the experimental $\mathrm{IC}_{50}$ values $\left(\mathrm{R}^{2}\right.$ $=0.62, P<0.0001$, Fig. 4A). Removal of the $\mathrm{Mg}^{2+}$ ion from the Mg3 site resulted in poor, nonsignificant correlation of the calculated binding energies with the experimental $\mathrm{IC}_{50}$ values $\left(\mathrm{R}^{2}=0.14, P=0.075\right.$, Fig. $\left.4 \mathrm{~B}\right)$. Thus, the structure of hGGPPS, solved in this study, is a highly useful model for

TABLE 2

Experimental and calculated binding parameters of bisphosphonates to hGGPPS

$\Delta \mathrm{G}$ values were calculated as described in Materials and Methods. The average $\Delta T_{\mathrm{m}}$ and $\mathrm{K}_{\mathrm{d}}$ values are shown as average \pm S.D., $n=6$ for all the experiments.

\begin{tabular}{|c|c|c|c|c|}
\hline \multirow{2}{*}{ Bisphosphonate } & \multirow{2}{*}{$\Delta T_{\mathrm{m}}$} & \multirow{2}{*}{$\mathrm{K}_{\mathrm{d}}(\mu \mathrm{M})$} & \multicolumn{2}{|c|}{$\underset{(\text { Kcal } / \mathrm{mol})}{\text { Calculated } \Delta \mathrm{G}}$} \\
\hline & & & $3 \mathrm{Mg}^{2+}$ & $2 \mathrm{Mg}^{2+}$ \\
\hline Ibandronate & $23.9 \pm 0.6$ & $1.74 \pm 0.22$ & 21.1 & 22.1 \\
\hline Zoledronate & $19.6 \pm 0.4$ & $2.76 \pm 0.31$ & 25.3 & 30.8 \\
\hline Alendronate & $16.0 \pm 0.2$ & $3.03 \pm 0.21$ & 30.3 & 36.2 \\
\hline Risedronate & $16.8 \pm 0.3$ & $3.35 \pm 0.30$ & 30.6 & 48.4 \\
\hline Neridronate & $17.4 \pm 0.3$ & $3.51 \pm 0.30$ & 32.1 & 31.5 \\
\hline Pamidronate & $15.8 \pm 0.3$ & $4.40 \pm 0.45$ & 34.1 & 33.3 \\
\hline
\end{tabular}

$T_{\mathrm{m}}$, melting temperature. computational drug-docking studies. Moreover, the presence of $\mathrm{Mg}^{2+}$ ions in all three sites is imperative for ligand-protein interactions, and their assignment is a crucial prerequisite for successful docking.

Reanalysis of yGGPPS-Bisphosphonate Complexes. We have previously reanalyzed the structure of the dimeric yGGPPS in complex with zoledronate (PDB 2E91) and were able to identify three $\mathrm{Mg}^{2+}$ ions in each monomer (Lisnyansky et al., 2018) with a coordination scheme similar to that reported in this study for hGGPPS in complex with ibandronate (Figs. 2B and 5A; Supplemental Material 2). Importantly, two yGGPPS structures, in complex with BPH-252 (PDB 2Z4X, Supplemental Material 3) and BPH-23 (PDB 2Z52, Supplemental Material 4), were originally assigned with three $\mathrm{Mg}^{2+}$ ions per monomer. Thus, we decided to re-examine additional deposited structures of yGGPPS (Supplemental Table 1) with a goal of identifying previously unassigned $\mathrm{Mg}^{2+}$ ions. Although the number of $\mathrm{Mg}^{2+}$ ions per monomer in the deposited structure varied between zero to three, we were able to assign three $\mathrm{Mg}^{2+}$ ions in all the re-refined structures (Supplemental Fig. 4; Table 4). The structural validity of this analysis is supported by improvement in the crystallographic statistics, with significant decrease in $R_{\text {free }}$, markedly improved model stereochemistry, and elimination of all the Ramachandran outliers in the deposited structures (Supplemental Table 1).

First, we analyzed deposited structures without any $\mathrm{Mg}^{2+}$ ions present. Given the surface electrostatics of the binding pocket (Fig. 2B), encompassing the aspartate-rich motifs, and of the phosphate groups, the absence of $\mathrm{Mg}^{2+}$ ions seemed highly unlikely. Indeed, although the original structures included no ions, we could position three $\mathrm{Mg}^{2+}$ ions in each chain (six ions in total) of yGGPPS-BPH806 (PDB 2Z78, Supplemental Material 5) and yGGPPS-BPH-sc01 (PDB 2Z4Z, Supplemental Material 6) (Supplemental Fig. 4). In these cases, the average $\mathrm{Mg}^{2+}-\mathrm{O}$ bond distances are $2.40 \pm$ $0.25 \AA$ (yGGPPS-BPH-806) and $2.23 \pm 0.25 \AA$ (yGGPPSBPH-sc01), and in each structure five $\mathrm{Mg}^{2+}$ ions have $\mathrm{CN} \geq 5$ and one ion has $\mathrm{CN}<5$ (Table 4).

Next, we analyzed deposited structures with two $\mathrm{Mg}^{2+}$ ions, similar to the case of yGGPPS-zoledronate (PDB 2E91). We have re-refined the structures of yGGPPS-minodronate (PDB 2E92, Supplemental Material 7) and yGGPPS-BPH-675 (PDB 2E95, Supplemental Material 8), with $\mathrm{Mg}^{2+}$ ions assigned only at position $\mathrm{Mg} 1$ and $\mathrm{Mg} 2$, leaving the Mg3 site vacant. For yGGPPS-minodronate, following addition of Mg3 to both chains, the average $\mathrm{Mg}^{2+}-\mathrm{O}$ bond distance was $2.17 \pm 0.13$ $\AA$, where three of the $\mathrm{Mg}^{2+}$ ions have $\mathrm{CN}=5$ and three $\mathrm{Mg}^{2+}$ ions have $\mathrm{CN}=4$ (Supplemental Fig. 4; Table 4). Similarly, following addition of Mg3 to both chains of yGGPPS-BPH-675, the average $\mathrm{Mg}^{2+}-\mathrm{O}$ bond distance is $2.14 \pm 0.12 \AA$, where all $\mathrm{Mg}^{2+}$ ions have $\mathrm{CN} \geq 5$ (Supplemental Fig. 4; Table 4).

Finally, we re-analyzed yGGPPS-BPH-210 (PDB 2Z7H, Supplemental Material 9), where $\mathrm{Mg}^{2+}$ binding differed between the chains: a single $\mathrm{Mg}^{2+}$ ion was assigned to one monomer and no ions were assigned to the second monomer. As in the abovementioned structures, we could readily add five additional ions in total, with three $\mathrm{Mg}^{2+}$ ions assigned to each chain (Supplemental Fig. 4). The average $\mathrm{Mg}^{2+}-\mathrm{O}$ bond distance is $2.24 \pm 0.23 \AA$, where all $\mathrm{Mg}^{2+}$ ions have $\mathrm{CN} \geq 5$ (Table 4). Overall, we could identify three $\mathrm{Mg}^{2+}$ ions per monomer in all of the re-refined structures presented in this 
A

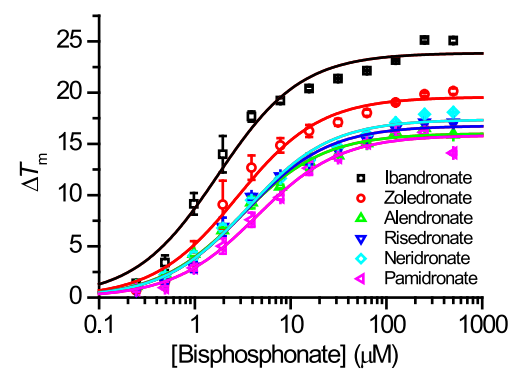

B

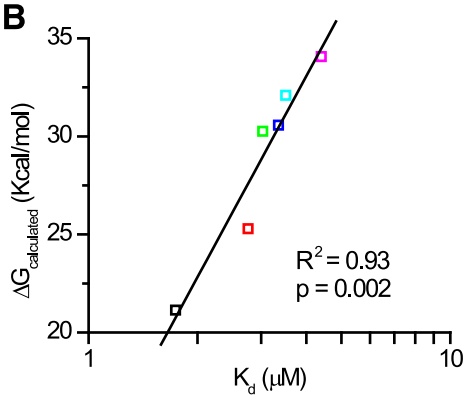

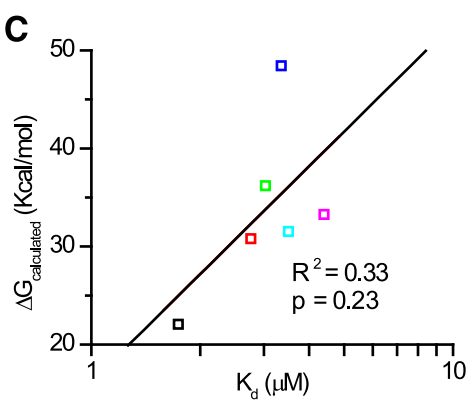

Fig. 3. Effect of $\mathrm{Mg}^{2+}$ stoichiometry on computational docking of commercial bisphosphonates to hGGPPS. (A) Differential scanning fluorimetry thermal shift assay in the presence of increasing bisphosphonate concentrations presented as $T_{\mathrm{m}}$ difference. Experiments were performed as outlined in Materials and Methods. Data are presented as mean \pm S.D., $n=6$. (B and C) Correlation between measured $\mathrm{K}_{\mathrm{d}}$ values (micromolar) and calculated binding energies (Kilocalorie per mole) obtained with $\mathrm{Mg}^{2+}$ ions occupying all three $\mathrm{Mg}^{2+}$ sites (B) or only sites $\mathrm{Mg} 1$ and $\mathrm{Mg} 2(\mathrm{C})$.

work (Table 4$)$, with an average $\mathrm{CN}=5.5 \pm 0.7$ (range $=3-6)$ and average $\mathrm{Mg}^{2+}-\mathrm{O}$ bond length $=2.18 \pm 0.20 \AA$, which closely match the expected values for $\mathrm{Mg}^{2+}$ ion coordination (Table 4). Thus, re-refinement of these structures uncovers a previously misidentified canonical binding scheme, demonstrating that, similar to hGGPPS, bisphosphonate binding to yGGPPS necessitates three $\mathrm{Mg}^{2+}$ ions (Supplemental Fig. 4; Table 4).

Effect of $\mathrm{Mg}^{2+}$ Ion Stoichiometry on Drug Docking to yGGPPS. To further validate the assignment of $\mathrm{Mg}^{2+}$ ions to the yGGPPS structures, and to generalize the functional significance of the metal-ligand stoichiometry, we performed additional virtual docking of bisphosphonates with known $\mathrm{K}_{\mathrm{i}}$ values toward yGGPPS (PDB 2E91) (Fig. 5; Table 5) (Guo et al., 2007). As in hGGPPS, to assess the contribution of Mg3, we performed this analysis with $\mathrm{Mg}^{2+}$ ions occupying all binding sites or only sites Mg1 and Mg2 (Table 5). Consistent with our crystallographic analysis (Fig. 5A; Supplemental Fig. 4), in the presence of all three $\mathrm{Mg}^{2+}$ ions, the calculated binding energies are highly correlated with the measured $\mathrm{K}_{\mathrm{i}}$ values $\left(\mathrm{R}^{2}=0.71, P=0.0042\right.$, Fig. $\left.5 \mathrm{~B}\right)$. However, leaving the Mg3 site vacant appreciably hindered the correlation between the calculated binding energies and the experimental $\mathrm{K}_{\mathrm{i}}$ values $\left(\mathrm{R}^{2}=0.44, P=0.052\right.$, Fig. $\left.5 \mathrm{C}\right)$. Thus, a three $\mathrm{Mg}^{2+}$ ion stoichiometry is common and essential for binding of bisphosphonates to both yGGPPS and hGGPPS.

\section{Discussion}

The goals of the present study were to elucidate the structural basis of bisphosphonates interaction with hGGPPS and to determine the accurate metal-ligand interaction network underlying GGPPS-bisphosphonate binding. In addition, given that mevalonate pathway enzymes, and specifically GGPPS, were highlighted in recent years as potential drug targets (Andela et al., 2003; Jiang et al., 2014; Gruenbacher and Thurnher, 2015), we sought to provide a reliable model for future drug design and screening. Importantly, preclinical studies have established the potential use of GGPPS inhibitors in the treatment of solid and hematologic malignancies, such as lung alveolar carcinoma (Andela et al., 2003), breast cancer (Ginestier et al., 2012), multiple myeloma (Lacbay et al., 2018), metastatic prostate cancer (Reilly et al., 2015, 2017), and KRAS-mutant lung cancer (Xia et al., 2014). Moreover, GGPPS enzymes from parasites leading to human diseases such as malaria, Chagas disease, leishmaniasis, and toxoplasmosis are considered as potential drug targets, as bisphosphonates were shown to inhibit parasitic growth in vitro and in vivo (Szabo et al., 2002; G Ricci et al., 2016). Collectively, these data establish the clinical potential of GGPPS inhibitors.

Despite the promising preclinical results of GGPPS inhibitors, bisphosphonates have several shortcomings, currently preventing their clinical use in the treatment of nonskeletal diseases. Their main disadvantages relate to their pharmacokinetics, with limited distribution focused mainly to the bone matrix (Lin, 1996). In addition, some bisphosphonates lack specificity toward GGPPS (Guo et al., 2007), and most of them display poor cell permeability (Haney et al., 2017). Therefore, the design of novel bisphosphonates with improved tissue distribution profiles, such as the lipophilic bisphosphonates (Zhang et al., 2009), bisphosphonates with improved GGPPS specificity (Zhou et al., 2014; Wills et al., 2015, 2017), and nonbisphosphonate GGPPS inhibitors, is highly desired (Merino et al., 2017). However, these translational efforts are hampered by the current ambiguity in metal-ligand interaction assignment within the binding site (Guo et al., 2007; K-M Chen et al., 2008; Zhang et al., 2009), a problem
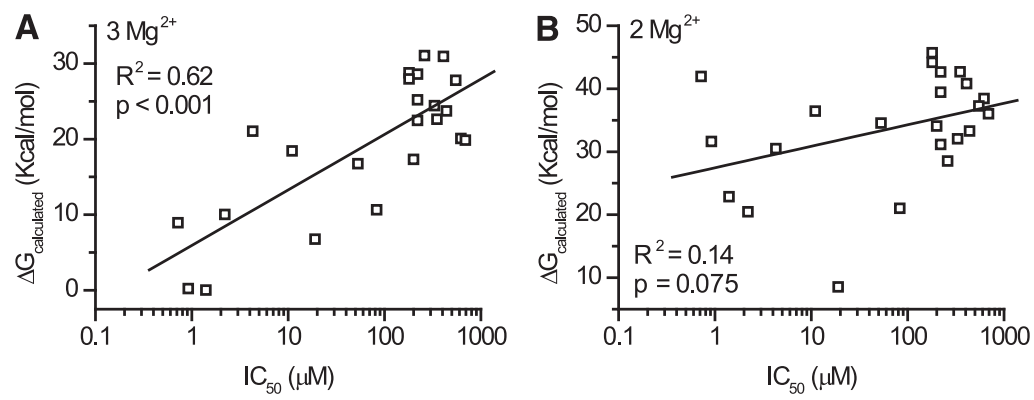

Fig. 4. Extended analysis of $\mathrm{Mg}^{2+}$ effect on computational docking of bisphosphonates to hGGPPS. (A and B) Correlation between measured $\mathrm{IC}_{50}$ values (micromolar) [reported by Szabo et al. (2002)] and calculated binding energies (Kilocalorie per mole) obtained with $\mathrm{Mg}^{2+}$ ions occupying all three $\mathrm{Mg}^{2+}$ sites (A) or only sites Mg1 and Mg2 (B). 


\section{TABLE 3}

Experimental $\mathrm{IC}_{50}$ values and calculated binding energies of bisphosphonates to hGGPPS

$\mathrm{IC}_{50}$ were obtained from Szabo et al. (2002). For full description of the compounds, please refer to the original publication. $\Delta \mathrm{G}$ values were calculated as described in Materials and Methods.

\begin{tabular}{|c|c|c|c|}
\hline \multirow{2}{*}{ Bisphosphonate } & \multirow{2}{*}{$\mathrm{IC}_{50}(\mu \mathrm{M})$} & \multicolumn{2}{|c|}{ Calculated $\Delta \mathrm{G}(\mathrm{Kcal} / \mathrm{mol})$} \\
\hline & & $3 \mathrm{Mg}^{2+}$ & $2 \mathrm{Mg}^{2+}$ \\
\hline 14 & 0.92 & 0.22 & 31.63 \\
\hline 15 & 1.4 & 0.02 & 22.84 \\
\hline 16 & 0.72 & 8.92 & 41.94 \\
\hline 17 & 4.3 & 21.05 & 30.49 \\
\hline 18 & 11 & 18.44 & 36.45 \\
\hline 19 & 53 & 16.74 & 34.55 \\
\hline 20 & 200 & 17.32 & 34.07 \\
\hline 21 & 620 & 20.09 & 38.45 \\
\hline 22 & 180 & 28.76 & 44.18 \\
\hline 23 & 220 & 25.19 & 42.65 \\
\hline 24 & 220 & 22.48 & 39.44 \\
\hline 25 & 220 & 28.58 & 31.15 \\
\hline 26 & 260 & 31.05 & 28.52 \\
\hline 27 & 350 & 22.63 & 42.69 \\
\hline 28 & 410 & 30.93 & 40.82 \\
\hline 29 & 550 & 27.78 & 37.25 \\
\hline 30 & 2.2 & 10.04 & 20.47 \\
\hline 31 & 19 & 6.77 & 8.52 \\
\hline 32 & 83 & 10.64 & 21.01 \\
\hline 33 & 330 & 24.42 & 32.05 \\
\hline 34 & 690 & 19.87 & 36.01 \\
\hline 35 & 440 & 23.72 & 33.29 \\
\hline 36 & 180 & 27.95 & 45.70 \\
\hline
\end{tabular}

common to other metalloenzymes as well (Puerta et al., 2003). This ambiguity impedes the ability of quantitative computational drug screening to predict protein-ligand interactions (Sommerhalter et al., 2005; Riccardi et al., 2018).

Although numerous yGGPPS structures in complex with different bisphosphonates are available, the affinity of these compounds toward hGGPPS and yGGPPS differs. For example, although the reported affinities of zoledronate and minodronate to yGGPPS are $~ 10$-fold higher compared with hGGPPS, and the affinity of BPH-364 to yGGPPS is 25-fold higher compared with hGGPPS, the affinity of other bisphosphonates such as BPH-628 and BPH-675 is practically identical for both orthologs (Guo et al., 2007). In this study, to provide a structural framework for the design of novel bisphosphonates for hGGPPS inhibition, we determined the first crystal structure of hGGPPS in complex with a bisphosphonate (Fig. 2). Our $2.2 \AA$ resolution crystal structure clearly resolved the hGGPPS-bisphosphonate binding mode, involving three $\mathrm{Mg}^{2+}$ ions. Rummaging through the Protein Data Bank (PDB), we found this binding mode only in two
yGGPPS-bisphosphosphonate complexes, but other metal stoichiometries ranging from zero to two $\mathrm{Mg}^{2+}$ ions were reported in most structures (of which we have reanalyzed six and assigned the canonical three $\mathrm{Mg}^{2+}$ ions) (Guo et al., 2007; K-M Chen et al., 2008; Zhang et al., 2009). Thus, we sought to determine whether the binding stoichiometry observed in this study for hGGPPS is a bona fide prerequisite for bisphosphonate binding, or if different bisphosphonates display different metal-ligand binding schemes.

Although X-ray crystallography is the gold standard for high-resolution structural investigations, it should be kept in mind that the reported crystal structures are only models that explain the electron density, and are subjected to interpretation by the crystallographer (Davis et al., 2003). The presence of metals, and specifically $\mathrm{Mg}^{2+}$, within ligand binding sites poses challenges in interpretation of the crystallographic data (Handing et al., 2018). First, $\mathrm{Mg}^{2+}$ has the same number of electrons as water, rendering them indistinguishable merely by their electron density. Thus, automatic assignment of water molecules to the model (Adams et al., 2010), developed to facilitate structure determination, may become a doubleedged sword by introducing bias and hampering the detection of other solvents such as $\mathrm{Mg}^{2+}, \mathrm{Na}^{+}$, or $\mathrm{NH}_{4}^{+}$(Davis et al., 2003), unless the added water molecules are critically examined. Moreover, the wavelength required to detect $\mathrm{Mg}^{2+}$ by anomalous diffraction, an approach commonly used to identify other metals in metalloenzymes, is outside the wavelength range obtainable in modern synchrotrons (Zheng et al., 2008, 2015; Harding et al., 2010). Indeed, previous studies of structures deposited in the PDB revealed misidentification of $\mathrm{Mg}^{2+}$ ions in complex with RNA (Zheng et al., 2015) or with metalloproteins (Zheng et al., 2008), including placement of $\mathrm{Mg}^{2+}$ instead of $\mathrm{Ca}^{2+}, \mathrm{Na}^{+}$, or water and vice versa. The incorrect placement of $\mathrm{Mg}^{2+}$ within the binding pocket of metalloenzymes has clear implications on subsequent computational drug screening and design. As we show in this study, the omission of a single $\mathrm{Mg}^{2+}$ ion from the bisphosphonatebinding scheme of either hGGPPS or yGGPPS results in poor model predictivity (Figs. 3-5). These results emphasize the importance of metal-ligand interactions in computational drug screening and design in GGPPS and other metalloenzymes in general.

Of note, the calculated $\Delta \mathrm{G}$ values were positive, which indicates that the ligands would not bind, in stark conflict with reality. A close examination of the energy decomposition indicates that this is due to an overestimate of the electrostatic desolvation component of the binding free energy (Kessel and Ben-Tal, 2018), caused by the numerous charges on both the ligands and protein binding site.

TABLE 4

$\mathrm{Mg}^{2+}$ coordination parameters in re-refined structures of yGGPPS

The average $\mathrm{Mg}^{2+}-\mathrm{O}$ bond length is shown as average \pm S.D.; numbers in parentheses indicate the sample size.

\begin{tabular}{|c|c|c|c|c|c|c|}
\hline Ligand & PDB & $\mathrm{Mg}^{2+} /$ Chain (Deposited) & $\mathrm{Mg}^{2+} /$ Chain (Re-refined) & $\mathrm{Mg}^{2+}-\mathrm{O}$ Bond Length $(\AA)$ & $\mathrm{CN} \geq 5$ & $\mathrm{CN}<5$ \\
\hline Zoledronate & 2E91 & 2 & 3 & $2.09 \pm 0.13(34)$ & 6 & 0 \\
\hline Minodronate & 2E92 & 2 & 3 & $2.17 \pm 0.13(27)$ & 3 & 3 \\
\hline BPH-675 & $2 \mathrm{E} 95$ & 2 & 3 & $2.14 \pm 0.12(35)$ & 6 & 0 \\
\hline ВРН-806 & $2 \mathrm{Z} 78$ & 0 & 3 & $2.40 \pm 0.25(31)$ & 5 & 1 \\
\hline BPH-sc01 & $2 \mathrm{Z} 4 \mathrm{Z}$ & 0 & 3 & $2.23 \pm 0.25(31)$ & 5 & 1 \\
\hline BPH-252 & $2 \mathrm{Z} 4 \mathrm{X}$ & 3 & 3 & $2.11 \pm 0.10(36)$ & 6 & 0 \\
\hline BPH-23 & $2 \mathrm{Z} 52$ & 3 & 3 & $2.10 \pm 0.17(36)$ & 6 & 0 \\
\hline
\end{tabular}



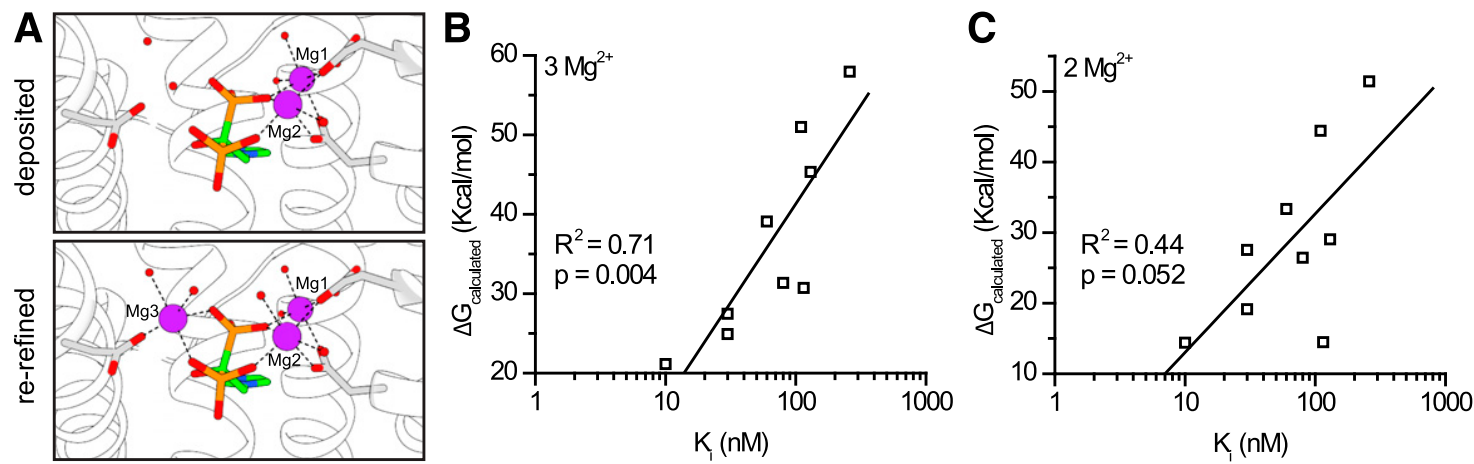

Fig. 5. Effect of $\mathrm{Mg}^{2+}$ stoichiometry on computational docking of bisphosphonates to yGGPPS. (A) Cartoon representations of the ligand-binding pocket in the deposited zoledronate-bound yGGPPS (PDB 2E91, upper panel) and the re-refined structure (lower panel). Zoledronate and residues interacting with the $\mathrm{Mg}^{2+}$ ions (purple spheres) are shown as sticks. Water molecules are shown as red spheres. (B and C) Correlation between reported $\mathrm{K}_{\mathrm{i}}$ values (nanomolar) and calculated binding energies (Kilocalorie per mole) obtained with $\mathrm{Mg}^{2+}$ ions occupying all three $\mathrm{Mg}^{2+}$ sites (B) or only sites $\mathrm{Mg} 1$ and Mg2 (C).

However, as all the molecules compared in this study share similar structure and as the protein structure changes vary little when binding different bisphosphonates (Guo et al., 2007), the error is subtracted out when comparing the relative values as observed in the slopes (Figs. 3-5), even though the absolute $\Delta \mathrm{G}$ values are consistently too positive.

In light of the computational docking results, which decisively favored three $\mathrm{Mg}^{2+}$ ions in the bisphosphonate coordination scheme (Figs. 3 and 4), we pursued a comprehensive reanalysis of previously deposited yGGPPS structures representing the four possible $\mathrm{Mg}^{2+}$ binding stoichiometries (Fig. 5). Using a set of stereochemical restraints, including bond lengths, identity of oxygen donors, and $\mathrm{CN}$, we could place three $\mathrm{Mg}^{2+}$ ions in all the re-refined structures presented in this study (Table 4). Importantly, the overall bonding parameters were as expected based on the Cambridge Structural Database and structures with similar resolution deposited in the PDB (Zheng et al., 2008). Together, our experimental and computational findings reiterate that $\mathrm{Mg}^{2+}$ ions should be carefully placed in crystallographic structures based on the binding site geometry.

In conclusion, using the combination of experimental and computational approaches, we have established the metal coordination network mediating bisphosphonate-GGPPS interactions. Our data underscore the importance of three $\mathrm{Mg}^{2+}$ ions form $\mathrm{drug}$ binding and their essential role in drug screening and design. This work provides a structural basis

\section{TABLE 5}

Experimental and calculated binding parameters of bisphosphonates to yGGPPS

$\mathrm{K}_{\mathrm{i}}$ values were obtained from Guo et al. (2007). For full description of the compounds, please refer to the original publication. $\Delta \mathrm{G}$ values were calculated as described in Materials and Methods.

\begin{tabular}{lccc}
\hline & & \multicolumn{2}{c}{ Calculated $\Delta \mathrm{G}(\mathrm{Kcal} /$} \\
mol)
\end{tabular}

for future rational design of additional MBP-harboring drugs targeting hGGPPS.

Supporting Information. There are crystallographic statistics for the re-refined yGGPPS structures (Supplemental Table 1), crystal packing (Supplemental Fig. 1) $\mathrm{Mg}^{2+}$ ions omit map (Supplemental Fig. 2), representative fluorescence-temperature relations of GGPPS in the presence of different ibandronate concentrations (Supplemental Fig. 3), comparison of the deposited, and re-refined yGGPPS-binding pockets (Supplemental Fig. 4). The structure of hGGPPS in complex with ibandronate and the re-refined yGGPPS structures are available as Supplemental Materials.

\section{Acknowledgments}

We thank the staff of ID30B at the European Synchrotron Radiation Facility for assistance with diffraction experimentation. This work was performed in partial fulfillment of the requirements for a $\mathrm{Ph} . \mathrm{D}$. degree of M.L. and a M.D. degree of O.S. (Sackler Faculty of Medicine, Tel Aviv University, Israel). We acknowledge the Edmund J. Safra Center for Bioinformatics at Tel Aviv for supporting E.Y.'s fellowship.

\section{Authorship Contributions}

Participated in research design: Giladi, Haitin.

Conducted experiments: Lisnyansky, Yariv, Segal, Marom.

Contributed new reagents or analytic tools: Loewenstein, Ben-Tal. Performed data analysis: Lisnyansky, Giladi, Haitin.

Wrote or contributed to the writing of the manuscript: Lisnyansky, Giladi, Haitin.

\section{References}

Adams PD, Afonine PV, Bunkóczi G, Chen VB, Davis IW, Echols N, Headd JJ, Hung LW, Kapral GJ, Grosse-Kunstleve RW, et al. (2010) PHENIX: a comprehensive Python-based system for macromolecular structure solution. Acta Crystallogr D Biol Crystallogr 66:213-221.

Andela VB, Pirri M, Schwarz EM, Puzas EJ, O'Keefe RJ, Rosenblatt JD, and Rosier RN (2003) The mevalonate synthesis pathway as a therapeutic target in cancer. Clin Orthop Relat Res 415S:S59-S66.

Bourne HR, Sanders DA, and McCormick F (1990) The GTPase superfamily: a conserved switch for diverse cell functions. Nature 348:125-132.

Bruno E, Buemi MR, Di Fiore A, De Luca L, Ferro S, Angeli A, Cirilli R, Sadutto D, Alterio V, Monti SM, et al. (2017) Probing molecular interactions between human carbonic anhydrases (hCAs) and a novel class of benzenesulfonamides. $J$ Med Chem 60:4316-4326.

Cohen SM (2017) A bioinorganic approach to fragment-based drug discovery targeting metalloenzymes. Acc Chem Res 50:2007-2016.

Davis AM, Teague SJ, and Kleywegt GJ (2003) Application and limitations of X-ray crystallographic data in structure-based ligand and drug design. Angew Chem Int Ed Engl 42:2718-2736.

Downward J (2003) Targeting RAS signalling pathways in cancer therapy. Nat Rev Cancer 3:11-22.

Emsley P and Cowtan K (2004) Coot: model-building tools for molecular graphics. Acta Crystallogr D Biol Crystallogr 60:2126-2132. 
Friesner RA, Banks JL, Murphy RB, Halgren TA, Klicic JJ, Mainz DT, Repasky MP, Knoll EH, Shelley M, Perry JK, et al. (2004) Glide: a new approach for rapid, accurate docking and scoring. 1. Method and assessment of docking accuracy. $J$ Med Chem 47:1739-1749.

Ginestier C, Monville F, Wicinski J, Cabaud O, Cervera N, Josselin E, Finetti P, Guille A, Larderet G, Viens P, et al. (2012) Mevalonate metabolism regulates basal breast cancer stem cells and is a potential therapeutic target. Stem Cells $\mathbf{3 0}$ : 1327-1337.

Greenwood JR, Calkins D, Sullivan AP, and Shelley JC (2010) Towards the comprehensive, rapid, and accurate prediction of the favorable tautomeric states of drug-like molecules in aqueous solution. J Comput Aided Mol Des 24:591-604.

G Ricci C, Liu YL, Zhang Y, Wang Y, Zhu W, Oldfield E, and McCammon JA (2016) Dynamic structure and inhibition of a malaria drug target: geranylgeranyl diphosphate synthase. Biochemistry 55:5180-5190.

Gruenbacher G and Thurnher M (2015) Mevalonate metabolism in cancer. Cancer Lett 356:192-196.

Guo R-T, Cao R, Liang P-H, Ko T-P, Chang T-H, Hudock MP, Jeng W-Y, Chen CK-M, Zhang Y, Song Y, et al. (2007) Bisphosphonates target multiple sites in both cisand trans-prenyltransferases. Proc Natl Acad Sci USA 104:10022-10027.

Halgren TA, Murphy RB, Friesner RA, Beard HS, Frye LL, Pollard WT, and Banks JL (2004) Glide: a new approach for rapid, accurate docking and scoring. 2. En richment factors in database screening. J Med Chem 47:1750-1759.

Handing KB, Niedzialkowska E, Shabalin IG, Kuhn ML, Zheng H, and Minor W (2018) Characterizing metal-binding sites in proteins with X-ray crystallography. Nat Protoc 13:1062-1090.

Haney SL, Wills VS, Wiemer DF, and Holstein SA (2017) Recent advances in the development of mammalian geranylgeranyl diphosphate synthase inhibitors. Molecules 22:E886.

Harder E, Damm W, Maple J, Wu C, Reboul M, Xiang JY, Wang L, Lupyan D, Dahlgren MK, Knight JL, et al. (2016) OPLS3: a force field providing broad coverage of drug-like small molecules and proteins. J Chem Theory Comput 12: $281-296$.

Harding MM, Nowicki MW, and Walkinshaw MD (2010) Metals in protein structures: a review of their principal features. Crystallogr Rev 16:247-302.

Jiang P, Mukthavaram R, Chao Y, Nomura N, Bharati IS, Fogal V, Pastorino S, Teng $\mathrm{D}$, Cong X, Pingle SC, et al. (2014) In vitro and in vivo anticancer effects of mevalonate pathway modulation on human cancer cells. $\mathrm{Br} J$ Cancer 111 $1562-1571$

Jiang Z, You Q, and Zhang X (2019) Medicinal chemistry of metal chelating fragments in metalloenzyme active sites: a perspective. Eur J Med Chem 165:172-197. Kabsch W (2010) XDS. Acta Crystallogr D Biol Crystallogr 66:125-132.

Kavanagh KL, Dunford JE, Bunkoczi G, Russell RGG, and Oppermann U (2006a) The crystal structure of human geranylgeranyl pyrophosphate synthase reveals a novel hexameric arrangement and inhibitory product binding. J Biol Chem $\mathbf{2 8 1}$ : 22004-22012.

Kavanagh KL, Guo K, Dunford JE, Wu X, Knapp S, Ebetino FH, Rogers MJ, Russell RGG, and Oppermann U (2006b) The molecular mechanism of nitrogen-containing bisphosphonates as antiosteoporosis drugs. Proc Natl Acad Sci USA 103: $7829-7834$

Kennard O (2015) Cambridge crystallographic database. Acta Crystallogr A 37:C343.

Kessel A and Ben-Tal N (2018) Introduction to Proteins: Structure, Function, and Motions, 2nd ed, Taylor \& Francis, Boca Raton, FL.

K-M Chen C, Hudock MP, Zhang Y, Guo RT, Cao R, No JH, Liang PH, Ko TP, Chang $\mathrm{TH}$, Chang SC, et al. (2008) Inhibition of geranylgeranyl diphosphate synthase by bisphosphonates: a crystallographic and computational investigation. J Med Chem 51:5594-5607.

Konstantinopoulos PA, Karamouzis MV, and Papavassiliou AG (2007) Posttranslational modifications and regulation of the RAS superfamily of GTPases as anticancer targets. Nat Rev Drug Discov 6:541-555.

Lacbay CM, Waller DD, Park J, Gómez Palou M, Vincent F, Huang XF, Ta V, Berghuis AM, Sebag M, and Tsantrizos YS (2018) Unraveling the prenylation-cancer paradox in multiple myeloma with novel geranylgeranyl pyrophosphate synthase (GGPPS) inhibitors. J Med Chem 61:6904-6917.

Li J, Abel R, Zhu K, Cao Y, Zhao S, and Friesner RA (2011) The VSGB 2.0 model a next generation energy model for high resolution protein structure modeling. Proteins 79:2794-2812.

Liang PH, Ko TP, and Wang AH (2002) Structure, mechanism and function of prenyltransferases. Eur J Biochem 269:3339-3354.

Lin JH (1996) Bisphosphonates: a review of their pharmacokinetic properties. Bone 18:75-85.

Lisnyansky M, Kapelushnik N, Ben-Bassat A, Marom M, Loewenstein A, Khananshvili D, Giladi M, and Haitin Y (2018) Reduced activity of geranylgeranyl diphosphate synthase mutant is involved in bisphosphonate-induced atypical fractures. Mol Pharmacol 94:1391-1400.
McCoy AJ, Grosse-Kunstleve RW, Adams PD, Winn MD, Storoni LC, and Read RJ (2007) Phaser crystallographic software. J Appl Cryst 40:658-674.

Merino P, Maiuolo L, Delso I, Algieri V, De Nino A, and Tejero T (2017) Chemical approaches to inhibitors of isoprenoid biosynthesis: targeting farnesyl and geranylgeranyl pyrophosphate synthases. RSC Adv 7:10947-10967.

Mullen PJ, Yu R, Longo J, Archer MC, and Penn LZ (2016) The interplay between cell signalling and the mevalonate pathway in cancer. Nat Rev Cancer 16:718-731.

Nayal M and Di Cera E (1996) Valence screening of water in protein crystals reveals potential $\mathrm{Na}+$ binding sites. J Mol Biol 256:228-234.

Niesen FH, Berglund H, and Vedadi M (2007) The use of differential scanning fluorimetry to detect ligand interactions that promote protein stability. Nat Protoc 2:2212-2221.

Olsson MH, Søndergaard CR, Rostkowski M, and Jensen JH (2011) PROPKA3: consistent treatment of internal and surface residues in empirical $\mathrm{pKa}$ predictions. $J$ Chem Theory Comput 7:525-537.

Puerta DT, Schames JR, Henchman RH, McCammon JA, and Cohen SM (2003) From model complexes to metalloprotein inhibition: a synergistic approach to structurebased drug discovery. Angew Chem Int Ed Engl 42:3772-3774.

Reilly JE, Neighbors JD, and Hohl RJ (2017) Targeting protein geranylgeranylation slows tumor development in a murine model of prostate cancer metastasis. Cancer Biol Ther 18:872-882.

Reilly JE, Neighbors JD, Tong H, Henry MD, and Hohl RJ (2015) Targeting geranylgeranylation reduces adrenal gland tumor burden in a murine model of prostate cancer metastasis. Clin Exp Metastasis 32:555-566.

Riccardi L, Genna V, and De Vivo M (2018) Metal-ligand interactions in drug design. Nat Rev Chem 2:100-112.

Russell RGG (2011) Bisphosphonates: the first 40 years. Bone 49:2-19.

Sastry GM, Adzhigirey M, Day T, Annabhimoju R, and Sherman W (2013) Protein and ligand preparation: parameters, protocols, and influence on virtual screening enrichments. J Comput Aided Mol Des 27:221-234.

Sommerhalter M, Lieberman RL, and Rosenzweig AC (2005) X-ray crystallography and biological metal centers: is seeing believing? Inorg Chem 44:770-778.

Søndergaard CR, Olsson MHM, Rostkowski M, and Jensen JH (2011) Improved treatment of ligands and coupling effects in empirical calculation and rationalization of pKa values. J Chem Theory Comput 7:2284-2295.

Strong M, Sawaya MR, Wang S, Phillips M, Cascio D, and Eisenberg D (2006) Toward the structural genomics of complexes: crystal structure of a PE/PPE protein complex from Mycobacterium tuberculosis. Proc Natl Acad Sci USA 103: $8060-8065$.

Szabo CM, Matsumura Y, Fukura S, Martin MB, Sanders JM, Sengupta S, Cieslak JA, Loftus TC, Lea CR, Lee HJ, et al. (2002) Inhibition of geranylgeranyl diphosphate synthase by bisphosphonates and diphosphates: a potential route to new bone antiresorption and antiparasitic agents. J Med Chem 45:2185-2196

Wang M and Casey PJ (2016) Protein prenylation: unique fats make their mark on biology. Nat Rev Mol Cell Biol 17:110-122.

Wills VS, Allen C, Holstein SA, and Wiemer DF (2015) Potent triazole bisphosphonate inhibitor of geranylgeranyl diphosphate synthase. ACS Med Chem Lett 6 : $1195-1198$

Wills VS, Metzger JI, Allen C, Varney ML, Wiemer DF, and Holstein SA (2017) Bishomoisoprenoid triazole bisphosphonates as inhibitors of geranylgeranyl diphosphate synthase. Bioorg Med Chem 25:2437-2444.

Xia Y, Liu YL, Xie Y, Zhu W, Guerra F, Shen S, Yeddula N, Fischer W, Low W, Zhou $\mathrm{X}$, et al. (2014) A combination therapy for KRAS-driven lung adenocarcinomas using lipophilic bisphosphonates and rapamycin. Sci Transl Med 6:263ra161.

Zhang Y, Cao R, Yin F, Hudock MP, Guo RT, Krysiak K, Mukherjee S, Gao YG, Robinson H, Song Y, et al. (2009) Lipophilic bisphosphonates as dual farnesyl/ geranylgeranyl diphosphate synthase inhibitors: an X-ray and NMR investigation. J Am Chem Soc 131:5153-5162.

Zheng H, Chruszcz M, Lasota P, Lebioda L, and Minor W (2008) Data mining of metal ion environments present in protein structures. J Inorg Biochem 102:1765-1776.

Zheng H, Shabalin IG, Handing KB, Bujnicki JM, and Minor W (2015) Magnesiumbinding architectures in RNA crystal structures: validation, binding preferences, classification and motif detection. Nucleic Acids Res 43:3789-3801.

Zhou X, Ferree SD, Wills VS, Born EJ, Tong H, Wiemer DF, and Holstein SA (2014) Geranyl and neryl triazole bisphosphonates as inhibitors of geranylgeranyl diphosphate synthase. Bioorg Med Chem 22:2791-2798.

Address correspondence to: Dr. Moshe Giladi, Department of Physiology and Pharmacology, Sackler Faculty of Medicine, Tel Aviv University, Tel Aviv 6997801, Israel. E-mail: moshegil@post.tau.ac.il; or Dr. Yoni Haitin, Department of Physiology and Pharmacology, Sackler Faculty of Medicine, Tel Aviv University, Tel Aviv 6997801, Israel. E-mail: yhaitin@post.tau.ac.il 